study utilized the theory of planned behavior to explore socio-cultural factors that influence condom use intentions among African migrant youth in South Africa.

Methods Using a qualitative research approach and snow-ball sampling procedures, indepth interviews were conducted with 20 migrant youth from other African countries, aged between 20-25 years and residing In Cape Town, South Africa.

Results Most African migrant youth residing in South Africa have acceptable levels of knowledge about condoms and condom use and linkages to HIV prevention and safer sexual practices. Traditional norms on sexual behaviour prohibit young migrants to freely discuss safer sexual choices. Gendered norms in sexual relationships, acculturation and cultural expectations of referent others reportedly influence intentions to use condom during sexual intercourse.

Conclusion The nexus among socio-cultural norms, acculturation and safer sexual choices are shown to be crucial to any HIV prevention interventions among African migrant youth in South Africa.

Disclosure No significant relationships.

\section{P109 PREVALENCE AND FACTORS ASSOCIATED WITH ANY DEPRESSIVE SYMPTOM AMONG HIV-INFECTED ADOLESCENTS IN THE REPUBLIC OF CONGO}

${ }^{1}$ Martin Herbas Ekat*, ${ }^{2}$ Marcel Yotebieng, ${ }^{3}$ Valériane Leroy. ${ }^{1}$ National AIDS Control Program, HIV Care Support Unit, Brazzaville, Congo; ${ }^{2}$ Ohio State University, Ohio, USA; ${ }^{3}$ INSERM Toulouse, Toulouse, USA

\subsection{6/sextrans-2019-sti.296}

Background To describe the frequency of any depressive symptom and associated factors among adolescents (10 - 19 years old) living with HIV in Brazzaville and Pointe Noire, Republic of Congo (RoC).

Methods Adolescents aged 10 to 19 years, on antiretroviral treatment, followed in the two Ambulatory Treatment Centers in Brazzaville and Pointe-Noire, RoC were included in this cross-sectional study. Between April 19 and July 9, 2018, faceto-face interviews were conducted with all participants using a standardized questionnaire that include the nine-item of the Patient Health Questionnaire (PHQ-9). Bivariate and multivariable log-binomial model were used to estimate the prevalence ratio (PR) and 95\% confidence interval $(95 \% \mathrm{CI})$ assessing the strength of the association between predictors and presence of depressive symptoms defined as PHQ-9 score $\geq 9$.

Results A total of 135 adolescents were interviewed. Overall, 67 (50\%) were male, 81(60\%) were 15-19 years old. PHQ-9 was $\geq 9$ among $52(39 \%)$ of participants. In bivariate analysis, the proportion of participants with PHQ-9 score $\geq 9$ was higher among participants who learnt about their HIV status after a prolonged illness; those who reported been sexually active, drinking beer, aged 15-19, stopped school, forget to take antiretroviral treatment more than 2 times in the 7 days preceding the interview, having lost both parents, and not having family support in taking ART and attending HIV care. In multivariable analysis, the following factors remained statistically significant: being 15-19 years old (PR:2.07;95\% CI:1.06-4.04), having stopped school (PR:1.60;95\%CI:1.062.42) and reporting instances of omission to take antiretroviral treatment more than 2 times in the 7 days preceding the interview (PR: 2.06;95\%CI:1.23-3.45).

Conclusion The prevalence of depressive symptoms among HIV-positive adolescents is high and is associated with older age, poor compliance, and dropping-out of school. Active screening for depression during routine medical visit and proper management is needed.

Disclosure No significant relationships.

\section{P113 RISK BEHAVIORS FOLLOWING HEPATITIS C TREATMENT AMONG GAY AND BISEXUAL MEN LIVING WITH HIV IN MELBOURNE, AUSTRALIA}

${ }^{1}$ Brendan Harney*, ${ }^{1}$ Mark Stoové, ${ }^{1}$ Rachel Sacks-Davis, 'Daniela Van Santen, ${ }^{2}$ Christopher Fairley, ${ }^{2}$ Nicholas Medland, ${ }^{3}$ Mark O'Reilly, ${ }^{4}$ Richard Moore, ${ }^{5}$ Bk Tee, ${ }^{6}$ Joseph Sasadeusz, ${ }^{7}$ David Iser, ${ }^{7}$ Janine Roney, ${ }^{8}$ Gail Matthews, ${ }^{9}$ Ed Gane, ${ }^{10}$ Maria Prins, ${ }^{1}$ Margaret Hellard, ${ }^{7}$ Joseph Doyle. 'Burnet Institute, Disease Elimination Program, Melbourne, Australia; ${ }^{2}$ Melbourne Sexual Health Centre, Melbourne, Australia; ${ }^{3}$ Prahran Market Clinic, Prahran, Australia; ${ }^{4}$ Northside Clinic, Fitzroy North, Australia; ${ }^{5}$ Centre Clinic, St Kilda, Australia; ${ }^{6}$ Royal Melbourne Hospital, Parkville, Australia; ${ }^{7}$ Alfred Health and Monash University, Department of Infectious Diseases, Melbourne, Australia; ${ }^{8}$ Kirby Institute, Sydney, Australia; ${ }^{9}$ Auckland City Hospital, Auckland, New Zealand; ${ }^{10}$ Public Health Service of Amsterdam, Amsterdam, Netherlands

\subsection{6/sextrans-2019-sti.297}

Background Hepatitis C virus (HCV) elimination among gay and bisexual men (GBM) living with HIV is feasible in many high-income countries. There is concern that risk behaviours following treatment may lead to reinfection and adversely impact HCV elimination goals. We examined risk behaviours prior to and following HCV treatment commencement among sexually active GBM living with HIV.

Methods Data were drawn from co-EC, a prospective study aiming to treat and eliminate $\mathrm{HCV}$ among people living with HIV. Pre and post-HCV treatment commencement changes in self-reported sexual and injecting drug-related behaviours among sexually active GBM attending primary and tertiary care clinics in Melbourne were assessed using McNemar's test. Modified Poisson regression with robust variance was used to examine factors associated with risk behaviours following treatment commencement.

Results Of 120 males who completed both a pre and posttreatment commencement questionnaire, 90 reported $\geq 1$ male sex partner before or after treatment commencement. Among these 90 sexually active GBM, there was no significant change pre- to post-treatment in condom-less anal intercourse with casual partners $(52.5 \% / 56.6 \%, p 0.513)$ or injecting drug use (41.2\%/45.9\%, $p$ 0.344), but a significant decrease in group $\operatorname{sex}(34.4 \% / 21.1 \%, p$ 0.011). Post-treatment commencement, condom-less intercourse (adjusted prevalence ratio (aPR) 1.80, $95 \% \mathrm{CI} 1.07-3.03, p 0.026)$ and group sex (aPR 4.53, 95\%CI 1.76-11.67, $p$ 0.002) was highest amongst those who had reported these behaviours pre-treatment. Post-treatment commencement, injecting drug use was associated with the use of crystal methamphetamine during follow-up (aPR 4.36, 95\%CI 1.27-14.94, p 0.019).

Conclusion HCV-related risk behaviours were common among sexually active GBM before and after HCV treatment and primarily occurred among the same men. There was no significant evidence of increasing risk behaviour following treatment. More frequent post-treatment $\mathrm{HCV}$ testing may be justified among GBM engaging in these behaviours to identify potential $\mathrm{HCV}$ reinfection and provide prompt re-treatment to prevent further transmission.

Disclosure No significant relationships. 